PROCEEDINGS OF THE

AMERICAN MATHEMATICAL SOCIETY

Volume 127, Number 8, Pages 2265-2271

S 0002-9939(99)04953-9

Article electronically published on March 23, 1999

\title{
AN EXTENSION OF H. CARTAN'S THEOREM
}

\author{
SO-CHIN CHEN AND SHIH-BIAU JANG
}

(Communicated by Steven R. Bell)

\begin{abstract}
In this article we prove that if $D \subset \mathbb{C}^{n}, n \geq 2$, is a bounded pseudoconvex domain with real analytic boundary, then for each $g(z) \in \operatorname{Aut}(D)$, there exists a fixed open neighborhood $\Omega_{g}$ of $\bar{D}$ and an open neighborhood $V_{g}$ of $g(z)$ in $\operatorname{Aut}(D)$ such that any $h(z) \in V_{g}$ can be extended holomorphically to $\Omega_{g}$, and that the action defined by

$$
\begin{aligned}
\pi: V_{g} \times \Omega_{g} & \rightarrow \mathbb{C}^{n} \\
(f, z) & \mapsto \pi(f, z)=f(z)
\end{aligned}
$$

is real analytic in joint variables. This extends H. Cartan's theorem beyond
\end{abstract} the boundary. Some applications are also discussed here.

\section{INTRODUCTION}

Let $D$ be a bounded domain in $\mathbb{C}^{n}$ with $n \geq 1$, and let $\operatorname{Aut}(D)$ be the automorphism group associated with $D$. It was shown by $\mathrm{H}$. Cartan that if $\operatorname{Aut}(D)$ is equipped with the compact-open topology, then $\operatorname{Aut}(D)$ becomes a real Lie group, and the action defined by

$$
\begin{array}{r}
\pi: \operatorname{Aut}(D) \times D \rightarrow D, \\
(f, z) \mapsto f(z),
\end{array}
$$

is real analytic in joint variables. For the details of the proof the reader is referred to Narasimhan [9]. Here the compact-open topology is equivalent to the topology induced by the uniform convergence on compact subsets of $D$, and it is known that any real Lie group automatically carries a real analytic structure on the underlying manifold.

It is remarkable to note that in Cartan's theorem no hypothesis on the smoothness of the boundary is assumed. In this article we are mainly concerned about the boundary behavior of the automorphisms. To answer such a question we need some smoothness condition on the boundary. Therefore, from now on we shall always assume that the domain $D$ has real analytic boundary $b D$ and that either $D$ is pseudoconvex or condition $R$ holds on $D$. By condition $R$ we mean that the Bergman projection $P$, the orthogonal projection from $L^{2}(D)$ onto the closed subspace $\mathcal{O}^{2}(D)$ of square integrable holomorphic functions, maps $C^{\infty}(\bar{D})$ continuously

Received by the editors October 21, 1997.

1991 Mathematics Subject Classification. Primary 32M05.

Key words and phrases. Automorphism group, pseudoconvex domains, condition $R$.

Both authors are partially supported by a grant NSC 85-2121-M-007-028 from the National Science Council of the Republic of China. 
into itself. Then the following fundamental theorem concerning the holomorphic extension of a smooth $C R$ diffeomorphism is proved by Baouendi, Jacobowitz and Treves in [1].

Theorem 1.1. Let $D \subset \mathbb{C}^{n}, n \geq 2$, be a smoothly bounded domain with real analytic boundary. Suppose that either $D$ is pseudoconvex or condition $R$ holds on $D$; then any automorphism $f(z) \in A u t(D)$ can be extended holomorphically to some open neighborhood $\Omega$ of $\bar{D}$.

By employing Theorem 1.1 we shall prove the following main results which extend H. Cartan's theorem beyond the boundary of the domain.

Theorem 1.2. Let D be a domain satisfying the hypotheses stated in Theorem 1.1. Then

(1) for each $g(z) \in \operatorname{Aut}(D)$, there exist a fixed open neighborhood $\Omega_{g}$ of $\bar{D}$ and an open neighborhood $V_{g}$ of $g(z)$ in Aut $(D)$ such that any $h(z) \in V_{g}$ can be extended holomorphically to $\Omega_{g}$;

(2) the action defined by

$$
\begin{aligned}
\pi: V_{g} \times \Omega_{g} & \rightarrow \mathbb{C}^{n}, \\
\quad(f, z) & \mapsto \pi(f, z)=f(z),
\end{aligned}
$$

is real analytic in joint variables.

The authors are indebted to Nancy Stanton for bringing the reference [8] to their attention. One should also note that related results in the $C^{\infty}$ setting can be found in Barrett [2] and Bedford [3]. As an application of Theorem 1.2 we can show the uniform holomorphic extendibility of the whole automorphism group $\operatorname{Aut}(D)$ when $\operatorname{Aut}(D)$ is compact.

Theorem 1.3. Let $D$ be a domain satisfying the hypotheses stated in Theorem 1.1. Then the following statements (1) and (2) are equivalent.

(1) Aut $(D)$ is compact.

(2) There exist a fixed open neighborhood $\Omega$ of $\bar{D}$ and a fixed constant $M>0$ such that each $g(z) \in \operatorname{Aut}(D)$ can be extended holomorphically to $\Omega$ and that $\sup _{\Omega}|g(z)| \leq M$.

The constant $M$ and the uniform boundedness condition for the automorphisms seem to be superfluous. This is true when $n=2$, according to the characterization of the domains with noncompact automorphism group due to Bedford and Pinchuk [4]. In general, the situation is still not clear. We conjecture that both statements in Theorem 1.3 should be equivalent to the following:

(3) There exists a fixed open neighborhood $\Omega$ of $\bar{D}$ such that every $g(z) \in \operatorname{Aut}(D)$ extends holomorphically to $\Omega$.

Some related works concerning the uniform holomorphic extendibility of the automorphisms across the boundary have been done on strictly pseudoconvex domains with real analytic boundaries. For instance, see Bell [5], Coupet [6], Krantz [7] and Vitushkin [10].

The following theorem is an immediate consequence of Theorems 1.2 and 1.3.

Theorem 1.4. Let $D$ be a domain satisfying the hypotheses stated in Theorem 1.1. Suppose that $\operatorname{Aut}(D)$ is compact. Then there exists a fixed open neighborhood $\Omega$ of 
$\bar{D}$ such that every automorphism $f(z) \in A u t(D)$ extends holomorphically to $\Omega$ and that the action,

$$
\begin{aligned}
\pi: \operatorname{Aut}(D) \times \Omega & \rightarrow \mathbb{C}^{n}, \\
(f, z) & \mapsto f(z),
\end{aligned}
$$

is real analytic in joint variables.

\section{Proof of the MAIN THEOREMS}

In this section we will prove Theorems 1.2 and 1.3. First we recall the Vitali's theorem which is needed in the proof.

Definition 2.1. A subset $E$ of a connected open set $\Omega \subseteq \mathbb{C}^{n}$ is called a set of uniqueness if any holomorphic function $f(z)$ on $\Omega$ vanishing on $E$ is identically zero.

For example, any nonempty open subset of $\Omega$ is a set of uniqueness by the identity theorem. Then we have

Theorem (Vitali). Let $\left\{f_{\nu}(z)\right\}$ be a sequence of holomorphic functions on the connected open set $\Omega$, and let $E$ be a set of uniqueness in $\Omega$. Suppose that $\left\{f_{\nu}(z)\right\}$ is uniformly bounded on $\Omega$, i.e., there exists an $M>0$ such that $\left|f_{\nu}(z)\right| \leq M$ for all $\nu$ and all $z \in \Omega$, and that $\left\{f_{\nu}(a)\right\}$ converges for any $a \in E$. Then $\left\{f_{\nu}(z)\right\}$ converges uniformly on any compact subsets of $\Omega$.

For the details of the proof the reader is referred to Narasimhan [9]. Now we proceed to prove (1) of Theorem 1.2. By Theorem 1.1, it suffices to prove the theorem for $g(z)=e=$ identity mapping in $\operatorname{Aut}(D)$. Assume that $\operatorname{dim}_{\mathbb{R}} \operatorname{Aut}(D)=k$. Consider a small open neighborhood $V$ of $e$ in $\operatorname{Aut}(D)$, and identify $V$ with an open neighborhood $V$ (we use the same notations) of the origin in $\mathbb{R}^{k}$. Hence $\bar{V}$ is a complete metric space.

Next denote by

$$
\Omega_{j}=\left\{z \in \mathbb{C}^{n} \mid \operatorname{dist}(z, \bar{D})<\frac{1}{j}\right\}, \text { for } j \in \mathbb{N},
$$

and we have $\bar{D}=\bigcap_{j=1}^{\infty} \Omega_{j}$. Now we see by Theorem 1.1 that for each $t \in \bar{V}$, the corresponding automorphism $g_{t}(z) \in \operatorname{Aut}(D)$ can be extended holomorphically to some open neighborhood $\Omega$ containing $\bar{D}$. Hence we have $g_{t}(z) \in H\left(\Omega_{j}\right)$ for some $j \in \mathbb{N}$. Put

$$
E_{j, m}=\left\{t \in \bar{V} \mid g_{t}(z) \in H\left(\Omega_{j}\right) \text { and } \sup _{z \in \Omega_{j}}\left|g_{t}(z)\right| \leq m\right\} .
$$

We obtain that

$$
\bar{V}=\bigcup_{j, m=1}^{\infty} E_{j, m}
$$

Next we show that $E_{j, m}$ is closed for each $(j, m) \in \mathbb{N} \times \mathbb{N}$. If $\left\{t_{\alpha}\right\}_{\alpha=1}^{\infty}$ is a Cauchy sequence in $E_{j, m}$ such that $\lim _{\alpha \rightarrow \infty} t_{\alpha}=t \in \bar{V}$, then we have $\left\{g_{t_{\alpha}}(z)\right\}$ converges uniformly on compact subsets of $D$ to $g_{t}(z)$ by H. Cartan's theorem, i.e., the mapping (1.1). Since $g_{t_{\alpha}}(z) \in H\left(\Omega_{j}\right)$ are uniformly bounded by $m$ on $\Omega_{j}$ for all $\alpha$, it follows 
by Vitali's theorem that $g_{t}(z) \in H\left(\Omega_{j}\right)$ and $\sup _{z \in \Omega_{j}}\left|g_{t}(z)\right| \leq m$ which shows that $t \in E_{j, m}$. So $E_{j, m}$ is a closed subset.

So now we can apply Baire's category theorem to conclude that some $E_{j, m}$ must contain an open subset $V_{0}$ of $V$. Hence after by composing $V_{0}$ with the inverse of $g_{t}(z)$ for some $t \in V_{0}$, we have shown that there exist an open neighborhood $V_{e}$ of $e$ in $\operatorname{Aut}(D)$, and a fixed open neighborhood $\Omega_{e}$ containing $\bar{D}$ and a fixed constant $M>0$ such that

$$
g_{t}(z) \in H\left(\Omega_{e}\right) \text { and } \sup _{z \in \Omega_{e}}\left|g_{t}(z)\right| \leq M,
$$

for any $g_{t}(z) \in V_{e}$. This completes the proof of (1) of Theorem 1.2.

To prove (2) of Theorem 1.2 we first show that the mapping (1.2) is continuous in joint variables. We observe that if $\left(t_{j}, z_{j}\right) \in V_{g} \times \Omega_{g}$ for $j \in \mathbb{N}$ is such that $\lim _{j \rightarrow \infty}\left(t_{j}, z_{j}\right)=(t, z) \in V_{g} \times \Omega_{g}$, we have

$$
\left|g_{t_{j}}\left(z_{j}\right)-g_{t}(z)\right| \leq\left|g_{t_{j}}\left(z_{j}\right)-g_{t}\left(z_{j}\right)\right|+\left|g_{t}\left(z_{j}\right)-g_{t}(z)\right| .
$$

The first term can be made arbitrarily small by Vitali's theorem if $z_{j}$ belongs to a small neighborhood of $z$ and $t_{j}$ is sufficiently close to $t$. The second term is also small because $g_{t}(z) \in H\left(\Omega_{g}\right)$. This shows that the mapping (1.2) is continuous in joint variables. By applying Cauchy's integral formula, we thus obtain

Corollary 2.2. For any multiindex $\alpha, \frac{\partial^{\alpha}}{\partial z^{\alpha}} g_{t}(z)$ is continuous on $V_{e} \times \Omega_{e}$.

Now, a standard argument from the theory of topological transformation groups can be adopted to show that the mapping (1.2) in fact is real analytic in joint variables. For instance, see Chapter 5 of [8]. For the self-containedness of the paper, we shall sketch the proof here. From Corollary 2.2 we may make the following choices.

(1) Let $V_{e}^{\prime}$ and $V_{e}^{\prime \prime}$ be open balls centered at the origin in $\mathbb{R}^{k}$ with $V_{e}^{\prime \prime} \subset V_{e}^{\prime} \subset V_{e}$, and let $\Omega_{e}^{\prime}$ and $\Omega_{e}^{\prime \prime}$ be open neighborhoods of $\bar{D}$ with $\Omega_{e}^{\prime \prime} \subset \Omega_{e}^{\prime} \subset \Omega_{e}$.

(2) We have $g_{t}(z) \in \Omega_{e}$ for $(t, z) \in \overline{V_{e}^{\prime}} \times \overline{\Omega_{e}^{\prime}}$, and $g_{t}(z) \in \Omega_{e}^{\prime}$ for $(t, z) \in \overline{V_{e}^{\prime \prime}} \times \overline{\Omega_{e}^{\prime \prime}}$.

(3) For any $t_{1}, t_{2} \in V_{e}^{\prime}$, we have $g_{t_{2}} \circ g_{t_{1}}=g_{t}$ for some $t \in V_{e}$. Similarly, if $t_{1}, t_{2} \in V_{e}^{\prime \prime}$, we have $g_{t_{2}} \circ g_{t_{1}}=g_{t}$ for some $t \in V_{e}^{\prime}$.

Under the above setting we have

Lemma 2.3. For $z_{0} \in \Omega_{e}^{\prime}$, there exists an open neighborhood $V_{z_{0}}$ of the identity mapping e contained in $V_{e}^{\prime}$ such that the power series expansions of $g_{t}(z)$ at $z_{0}$ for all $t \in V_{z_{0}}$ are uniformly dominated by a convergent power series.

Proof. The assertion is an easy consequence of the Baire's category argument. So we omit it.

The following theorem is the key for proving the real analyticity of the mapping (1.2).

Theorem 2.4. On $V_{e}^{\prime} \times \Omega_{e}^{\prime}, \frac{\partial}{\partial t_{j}} g_{t}^{i}(z)$ exists and is simultaneously continuous in $(t, z)$, where $g_{t}(z)=\left(g_{t}^{1}(z), \ldots, g_{t}^{n}(z)\right)$ and $t=\left(t_{1}, \ldots, t_{k}\right)$. In particular, $g_{t}(z)$ is of class $C^{1}$ on $V_{e}^{\prime} \times \Omega_{e}^{\prime}$ with respect to $(t, z)$.

The proof of Theorem 2.4 will be done in real setting. Hence, a point $z \in \Omega_{e}$ will sometimes be denoted by $x$. Let $b \in V_{e}$ with $|b|=\eta$ for some fixed small 
$\eta>0$. Denote by $f(s, b, x)=g_{s b}(x)$ the one parameter subgroup. We write $f=\left(f_{1}+i f_{2}, \ldots, f_{2 n-1}+i f_{2 n}\right)$ and $z_{j}=x_{2 j-1}+i x_{2 j}$ for $1 \leq j \leq n$.

Lemma 2.5. For fixed $x \in \Omega_{e}$, the derivatives $\frac{\partial}{\partial s} f_{i}(0, b, x)$ for $1 \leq i \leq 2 n$ exist and are continuous on $\left\{b \in V_{e} \| b \mid=\eta\right\} \times \Omega_{e}$, and for small $h=h_{x}$ we have

$$
f_{i}(h, b, x)-f_{i}(0, b, x)=\sum_{j=1}^{2 n}\left(\int_{0}^{h} \frac{\partial}{\partial x_{j}} f_{i}(s, b, x) d s\right)\left(\frac{\partial}{\partial s} f_{j}(0, b, x)\right) .
$$

Proof. Let $\epsilon$ be a small positive number, and let $y=f(\epsilon, b, x)$, we get

$$
\begin{aligned}
\int_{0}^{h} f_{i}(s, b, y) d s-\int_{0}^{h} f_{i}(s, b, x) d s & =\int_{0}^{h} f_{i}(s+\epsilon, b, x) d s-\int_{0}^{h} f_{i}(s, b, x) d s \\
& =\int_{\epsilon}^{h+\epsilon} f_{i}(s, b, x) d s-\int_{0}^{h} f_{i}(s, b, x) d s \\
& =\int_{0}^{\epsilon} f_{i}(h+s, b, x) d s-\int_{0}^{\epsilon} f_{i}(s, b, x) d s .
\end{aligned}
$$

On the other hand, by Corollary 2.2 and the mean value theorem we obtain

$$
\int_{0}^{h} f_{i}(s, b, y) d s-\int_{0}^{h} f_{i}(s, b, x) d s=\sum_{j=1}^{2 n}\left(\int_{0}^{h} \frac{\partial}{\partial x_{j}} f_{i}\left(s, b, \bar{x}_{i j}\right) d s\right)\left(y_{j}-x_{j}\right),
$$

where $\bar{x}_{i j}=\left(y_{1}, \ldots, y_{j-1}, x_{j}+\theta_{i j}\left(y_{j}-x_{j}\right), x_{j+1}, \ldots, x_{2 n}\right)$ with $0<\theta_{i j}<1$ for $1 \leq j \leq 2 n$. It follows that we have

$$
\frac{1}{\epsilon}\left(\int_{0}^{\epsilon} f_{i}(h+s, b, x) d s-\int_{0}^{\epsilon} f_{i}(s, b, x) d s\right)=\sum_{j=1}^{2 n}\left(\int_{0}^{h} \frac{\partial}{\partial x_{j}} f_{i}\left(s, b, \bar{x}_{i j}\right) d s\right) \frac{\left(y_{j}-x_{j}\right)}{\epsilon} .
$$

Note that the matrix

$$
\left(\frac{\partial}{\partial x_{j}} f_{i}\left(s, b, \bar{x}_{i j}\right)\right)_{i, j=1}^{2 n}
$$

is uniformly close to the identity matrix for $s$ near zero and $\bar{x}_{i j}$ in some compact neighborhood of $x$. Thus, the matrix (2.3) is uniformly invertible. By letting $\epsilon$ tend to zero, we obtain (2.2). Now, the continuity of $\frac{\partial}{\partial s} f_{i}(0, b, x)$ for $1 \leq i \leq 2 n$ on $\left\{b \in V_{e}|| b \mid=\eta\right\} \times \Omega_{e}$ is an easy consequence of (2.2). This proves Lemma 2.5.

Lemma 2.6. On $V_{e}^{\prime} \times \Omega_{e}^{\prime}$, the partial derivatives

$$
\frac{\partial}{\partial t_{j}} g_{t}(z) \quad \text { for } \quad 1 \leq j \leq k
$$

exist and are continuous in $(t, z)$.

Proof. As before we shall denote the point by $x$, and it suffices to prove the lemma for $t_{1}$. Let $t=\left(t_{1}, \ldots, t_{k}\right) \in V_{e}^{\prime}$, and let $h$ be a small number so that $t^{\prime}=$ $\left(t_{1}+h, t_{2}, \ldots, t_{k}\right) \in V_{e}^{\prime}$. Define $\epsilon(t, h)$ by $g_{t^{\prime}} \circ g_{t}^{-1}=g_{\epsilon(t, h)}$. Thus, one may write $\epsilon=\epsilon(t, h)$ as

$$
\begin{aligned}
& \epsilon_{1}(t, h)=h+\delta(t, h), \\
& \epsilon_{j}(t, h)=\delta(t, h), \quad \text { for } \quad 2 \leq j \leq k,
\end{aligned}
$$


where $\delta(t, h)=\mathcal{O}\left(|(t, h)|^{2}\right)$ is divisible by $h$. Then we normalize $\epsilon(t, h)$ as

$$
\epsilon(t, h)=b(t, h) \cdot s(t, h)
$$

so that both $b(t, h)=\left(b_{1}(t, h), \ldots, b_{k}(t, h)\right)$ and $s(t, h) \in \mathbb{R}$ are real analytic in $(t, h)$ with $|b(t, h)|=\eta$ and that $b_{1}(t, h)=\eta+\mathcal{O}(|(t, h)|)$.

Now, by Lemma 2.5 we have

$$
\begin{aligned}
& \frac{1}{h}\left(g_{t^{\prime}}(x)-g_{t}(x)\right) \\
& =\frac{1}{h}\left(g_{\epsilon}\left(g_{t}(x)\right)-g_{0}\left(g_{t}(x)\right)\right) \\
& =\frac{1}{h}\left(f\left(s(t, h), b(t, h), g_{t}(x)\right)-f\left(0, b(t, h), g_{t}(x)\right)\right) \\
& =\sum_{j=1}^{2 n}\left(\frac{1}{h} \int_{0}^{s(t, h)} \frac{\partial}{\partial x_{j}} f\left(\tau, b(t, h), g_{t}(x)\right) d \tau\right)\left(\frac{\partial}{\partial s} f_{j}\left(0, b(t, h), g_{t}(x)\right)\right) .
\end{aligned}
$$

Letting $h$ tend to zero, we obtain

$$
\begin{aligned}
& \lim _{h \rightarrow 0} \frac{1}{h}\left(g_{t^{\prime}}(x)-g_{t}(x)\right) \\
& =\frac{\partial s}{\partial h}(t, 0) \sum_{j=1}^{2 n} \frac{\partial}{\partial x_{j}} f\left(0, b(t, 0), g_{t}(x)\right)\left(\frac{\partial}{\partial s} f_{j}\left(0, b(t, 0), g_{t}(x)\right)\right) .
\end{aligned}
$$

By Lemma 2.5 the proof of Lemma 2.6 is now complete. This also proves the Theorem 2.4.

If we let $b=(\eta, 0, \ldots, 0)$ in $(2.2)$, it is easily seen by Lemma 2.3 that we have

Lemma 2.7. The derivatives

$$
\left.\frac{\partial}{\partial t_{j}} g_{t}(z)\right|_{t=0} \quad \text { for } \quad 1 \leq j \leq k
$$

are real analytic in $z$ on $\Omega_{e}^{\prime}$.

Now, we proceed to finish the proof of (2) of Theorem 1.2. Consider the independent variables $t, \alpha \in V_{e}^{\prime \prime}$ and $z \in \Omega_{e}^{\prime \prime}$. Let $s \in V_{e}^{\prime}$ be such that $g_{s} \circ g_{t}=g_{\alpha}$. Let $x^{\prime}=g_{t}(x)$; then

$$
g_{s}\left(g_{t}(x)\right)=g_{\alpha}(x) .
$$

Differentiate $(2.4)$ with respect to $t_{j}$ for $1 \leq j \leq k$; we obtain

$$
\sum_{l=1}^{k} \frac{\partial g_{s}}{\partial s_{l}}\left(g_{t}(x)\right) \frac{\partial s_{l}}{\partial t_{j}}+\sum_{p=1}^{2 n} \frac{\partial g_{s}\left(g_{t}(x)\right)}{\partial x_{p}^{\prime}} \frac{\partial x_{p}^{\prime}}{\partial t_{j}}=0
$$

When $s=0$, the identity mapping, one can solve (2.5) for $\frac{\partial x_{p}^{\prime}}{\partial t_{j}}$ and get

$$
\frac{\partial g_{t}(x)}{\partial t_{j}}=-\left.\left.\sum_{l=1}^{k} \frac{\partial g_{s}}{\partial s_{l}}\left(g_{t}(x)\right)\right|_{s=0} \cdot \frac{\partial s_{l}}{\partial t_{j}}\right|_{s=0} .
$$

By Lemma 2.7 the right hand side is real analytic in $g_{t}(x)$. Hence, by the uniqueness from the theory of ordinary differential equation and the Cauchy- Kowalevski's theorem, we prove (2) of Theorem 1.2. 
Finally, we prove Theorem 1.3.

(1) implies (2). This follows immediately from the compactness of $\operatorname{Aut}(D)$ and the proof of (1) of the Theorem 1.2. So we are done.

(2) implies (1). If $\operatorname{Aut}(D)$ is not compact, then there exist a sequence of automorphisms $\left\{g_{j}(z)\right\}$ for $j \in \mathbb{N}$ and a point $w \in D$ such that $\lim _{j \rightarrow \infty} g_{j}(w)=p$ for some boundary point $p$. By passing to a subsequence, if necessary, we may also assume that the sequence of the inverse mapping $\left\{h_{j}(z)=g_{j}^{-1}(z)\right\}$ converges and satisfies $\lim _{j \rightarrow \infty} h_{j}(w)=q$ for a boundary point $q$. Hence we have

$$
g_{j} \circ h_{j}(z)=z \quad \text { and } \quad \operatorname{det}\left(g_{j}^{\prime}\left(h_{j}(w)\right)\right) \cdot \operatorname{det}\left(h_{j}^{\prime}(w)\right)=1 .
$$

Now by H. Cartan's theorem (see also Narasimhan [9]) we get

$$
\lim _{j \rightarrow \infty} \operatorname{det}\left(h_{j}^{\prime}(w)\right)=0 .
$$

It follows that

$$
\lim _{j \rightarrow \infty}\left(\sup _{z \in D}\left|\operatorname{det}\left(g_{j}^{\prime}(z)\right)\right|\right)=\infty .
$$

However, by assumption (2) and Vitali's theorem, we must have

$$
\lim _{j \rightarrow \infty}\left(\sup _{z \in D}\left|\operatorname{det}\left(g_{j}^{\prime}(z)\right)\right|\right)=0 .
$$

This gives the desired contradiction. Therefore, $\operatorname{Aut}(D)$ is compact, and the proof is now complete.

\section{REFERENCES}

1. M.S.Baouendi, H.Jacobowitz and F.Treves, On the analyticity of $C R$ mappings, Ann. of Math., 122 (1985), 365-400. MR 87f:32044

2. D.Barrett, Regularity of the Bergman projection on domains with transverse symmetries, Math. Ann., 258 (1982), 441-446. MR 83i:32032

3. E.Bedford, Action of the automorphisms of a smooth domain in $\mathbb{C}^{n}$, Proc. Amer. Math. Soc., 93, (No.2) (1985), 232-234. MR 86e:32029

4. E.Bedford and S.Pinchuk, Domains in $\mathbb{C}^{n+1}$ with noncompact automorphism group, J. of Geometric Analysis, 1, (No.3) (1991), 165-191. MR 92f:32024

5. S.Bell, Weakly pseudoconvex domains with noncompact automorphism groups, Math. Ann., 280 (1988), 403-408. MR 89h:32052

6. B.Coupet, Uniform extendibility of automorphisms, Contemporary Math. 137, Amer. Math. Soc., (1992), 177-183. MR 93j:32033

7. S.Krantz, Compactness principles in complex analysis, Seminarios I Volumen 3, Universidad autonóma de Madrid. División de Matematicas.

8. D. Montgomery and L.Zippin, Topological transformation groups, Wiley-Interscience, New York (1955). MR 17:383b

9. R.Narasimhan, Several complex variables, Univ. of Chicago Press, Chicago (1971). MR 49:7470

10. A.G. Vitushkin, Holomorphic continuation of maps of compact hypersurfaces, Math. USSR t.2v., 20 (1983), 27-33.

Department of Mathematics, National Tsing Hua University, Hsinchu, Taiwan, RePUBLIC OF CHINA 